\title{
Symmetry Energy Coefficients for Asymmetric Nuclear Matter
}

\author{
Fábio L. Braghin \\ Nuclear Theory and Elementary Particle Phenomenology Group, \\ Instituto de Física, Universidade de São Paulo, \\ C.P. 66.318, CEP 05315-970, São Paulo, SP, Brazil
}

Received on 30 October, 2002

\begin{abstract}
Symmetry energy coefficients of asymmetric nuclear matter generalized are investigated as the inverse of nuclear matter polarizabilities with two different approaches. Firstly a general calculation shows they may depend on the neutron-proton asymmetry itself. The choice of particular prescriptions for the density fluctuations lead to certain isospin (n-p asymmetry) dependences of the polarizabilities. Secondly, with Skyrme type interactions, the static limit of the dynamical polarizability is investigated corresponding to the inverse symmetry energy coefficient which assumes different values at different asymmetries (and densities and temperatures). The symmetry energy coefficient (in the isovector channel) is found to increase as n-p asymmetries increase. The spin symmetry energy coefficient is also briefly investigated.
\end{abstract}

\section{Introduction}

The (n-p) symmetry energy coefficient and its dependence on the nuclear density has been extensively studied and this is of relevance, for example, for the description of macroscopic nuclear properties as well as for proto-neutron and neutron stars. It represents the tendency of nuclear forces to have greater binding energies (E/A) for symmetric systems - equal number of protons and neutrons. It contributes as a coefficient for the squared neutron-proton asymmetry in usual macroscopic mass formula, $E / A=H_{0}(A, Z)+$ $a_{\tau}(N-Z)^{2} / A^{2}$, where $H_{0}$ does not depend on the asymmetry, Z, N and A are the proton, neutron and mass numbers respectively. Other powers of the asymmetry (proportional to $(N-Z)^{n}$ for $\left.n \neq 2[1]\right)$ are usually expected to be less relevant for the equation of state (EOS) of nuclear matter based on such parameterizations [2,3]. The same kind of parameterization is considered for nuclear matter where instead of nucleon numbers one has to deal with densities. $a_{\tau}$ is also the parameter which measures the response of the system to a perturbation which tends to separate protons from neutrons. It is given by the static polarizability of the system which also may depend on the asymmetry of the medium. This point has been developped and emphasized recently $[4,5]$. The spin symmetry energy coefficient of nuclear matter may also be defined, $a_{\sigma}$, representing the cost in energy to make the system spin-asymmetric (and eventually polarized nuclear matter). The spin channel is relevant for the study of the neutrino interaction with matter because it couples with axial vector current together with the scalar channel $[6,7,8]$. A suppression of the spin susceptibility (in this work we will be dealing rather with its inverse) leads to the suppression of Gamow Teller transitions which are of interest for the supernovae mechanism [8] and eventually to instabilities associated to ferromagnetic polarized states $[9,8]$. In this work we articulate and extend the ideas developped previously for the dependence of symmetry energy coefficients on neutron-proton asymmetry. For this we use a calculation for the static polarizabilities - proportional to the inverse of the symmetry coefficients in asymmetric matter which was done using Skyrme effective forces in [5, 4].

\section{Generalized Symmetry Energy Coefficients}

When considering a small amplitude external perturbation $\epsilon$, the medium polarizability is defined as the ratio of the density fluctuation $\left(\beta=\delta \rho_{n}-\delta \rho_{p}\right)$ to the amplitude of the external perturbation and it can be written as $[5,10]$ :

$$
\Pi^{s, t} \equiv \frac{\beta}{\epsilon}=-\frac{\rho}{2 \mathcal{A}_{s, t}(b, \beta)},
$$

where $\mathcal{A}_{s, t}$ is the neutron-proton (isovector) symmetry coefficient ( $s=0, t=1$ - spin, isospin) and $b=\rho_{n} / \rho_{p}-1$ is an asymmetry coefficient. For the other channels (different $(s, t)$ ) one may define different symmetry coefficients. Note that $\mathcal{A}_{s, t}$ is a function of $b$ and $\beta$ and these parameters may be related, as argued below. The occurrence of these functional dependences of $\mathcal{A}_{s, t}$ can be found just by the first 
stability condition with respect to the (density) fluctuation from which one defines the polarizability (1): $d H / d \beta=0$.

\section{A. Isospin dependence of $\mathcal{A}_{s, t}$}

We consider $\mathcal{A}_{s, t}$ to be a function of the density fluctuation $\beta$. Although $\beta$ is not the explicit $n-p$ asymmetry (given by $b$ ) we will consider that it depends on it (as it was also argued in [5]). We consider these parameters are related to each other and therefore we will write $\mathcal{A}=\mathcal{A}(\beta)$ shortly. In [4] two different prescriptions were discussed for $\beta$ in the calculation of the response function of asymmetric nuclear matter. We have used (and it was shown to be the more reasonable prescription) the one which leads to the following relation between the fluctuation $\beta$ and the explicit asymmetry $(b)$ :

$$
\beta=\delta \rho_{n}\left(\frac{2+b}{1+b}\right),
$$

Where $\delta \rho_{n}$ is the neutron density fluctuation. In the $n-p$ symmetric limit $\beta=2 \delta \rho_{n}$ and in another limit, in neutron matter, $\beta=\delta \rho_{n}$. The above prescription (expression (2)) is based on the assumption that the density fluctuations are proportional to the respective density of neutrons and protons, i.e., $\delta \rho_{n} / \beta=\rho_{n} / \rho$, being $\rho$ the total density. In spite of being rather well suited for the isovector channel, this kind of assumption can be considered as a starting point for the other channels (spin, scalar) in asymmetric nuclear matter. Prescription (2) is therefore model-dependent and different choices for it yield other forms for the the (asymmetric) static screening functions. The dynamic response functions are less sensitive to this prescription [4].
From the solution of the polarizability (1) we calculate the first derivative with relation to $b$ :

$$
\frac{d \beta}{d b}=\frac{\epsilon \rho}{2 \mathcal{A}_{0,1}^{2}} \frac{d \mathcal{A}^{0,1}}{d b}=-\frac{\beta}{\mathcal{A}_{0,1}} \frac{d \mathcal{A}^{0,1}}{d b} .
$$

Another expression can be obtained from the relation between $b$ and $\beta$ of (2). It yields:

$$
\frac{d \beta}{d b}=-\frac{\beta}{(2+b)(1+b)} .
$$

Equating these two last equations we obtain:

$$
-\mathcal{A}^{0,1} \frac{\beta}{(2+b)(1+b)}=-\beta \frac{d \mathcal{A}^{0,1}}{d b},
$$

From which it is possible to derive the following relation between the isospin s.e.c. and the n-p asymmetry [5]:

$$
\mathcal{A}^{0,1}=\mathcal{A}_{\text {sym }}^{0,1} \frac{2+2 b}{2+b} .
$$

In this expression $\mathcal{A}_{\text {sym }}=a_{\tau} \simeq 30 \mathrm{MeV}$ is the s.e.c. of symmetric nuclear matter $(b=0)$. For $b=2$ (neutron density three times larger than the proton density) we obtain $\mathcal{A}=1.5 \mathcal{A}_{\text {sym }}$. In the limit of neutron matter $\mathcal{A}(b \rightarrow \infty)=$ $2 \mathcal{A}_{\text {sym }}$.

Other prescriptions for the density fluctuation, leading to different dependences on the n-p asymmetry $(b)$, can be given by:

$$
\begin{aligned}
& \text { 1) } \beta=\text { const } \rightarrow \mathcal{A}_{0,1}=\text { const } \\
& \text { 2) } \beta=\delta \rho_{n} \frac{2+b^{m}}{1+b^{m}} \rightarrow \mathcal{A}_{0,1}=\mathcal{A}_{\text {sym }} \frac{2+2 b^{m}}{2+b^{m}}(m>1 \quad \text { integer }) .
\end{aligned}
$$

The first of these alternative prescriptions leads to a constant symmetry energy coefficient $\mathcal{A}=a_{\tau}$. However, it corresponds to $\delta \rho_{n}=\delta \rho_{p}$, independently of the asymmetry of the medium. This does not seems to be reasonable for example because in the limit of neutron matter there would be no proton density. Furthermore this prescription has been used in the dynamical response function yielding seemingly non physical results [4]. The second of prescriptions (7) is a very general one valid for arbitrary integer numbers $m$ and was considered for the sake of simple algebraic calculation. Probably $m$ should be not large because it would lead to a too much strong (stiff) dependence on $b$.

Another assumption for deriving expressions (6) and (7) was that $\rho$ is independent of $b$. This would be unreasonable if one considers a complete self consistent calculation with the equation of state of a proto-neutron star, for example.

\section{Polarizabilities with Skyrme forces}

A nearly exact expression for the dynamical polarizability of a non relativistic hot asymmetric nuclear matter at variable densities was derived with Skyrme interactions in [4].

The general static screening function $A_{s, t}$ in asymmetric nuclear matter at finite temperature was explicitely written in [5]. (The coefficient $b$ is related to a frequently used asymmetry coefficient: $\alpha=\left(2 \rho_{0 n}-\rho_{0}\right) / \rho_{0}$, by the expression: $b=2 \alpha /(1-\alpha)$.

\section{A. Results for Skyrme interactions}

The Skyrme interactions used are: SLyb [11], SkSC4, SkSC6 and SkSC10 [12, 13]. In Figure 1 we show the in- 
verse of the static polarizability (generalized symmetry energy coefficient) in the isovector channel for diverse Skyrme forces. as a function of the asymmetry parameter $b$. For symmetric nuclear matter $b=0$ the different Skyrme forces yield values between 27 and $34 \mathrm{MeV}$, the usual values adopted in the litterature. For increasing $b$ the coefficient increases, being the slope strongly dependent on the interaction. We plot one case for dense nuclear matter $\rho=2 \rho_{0}$ (long dashed-short dashed line) with force SLy(b). We note that the increase of $A_{0,1}$ is much smaller. These results were discussed more extensively in [5].

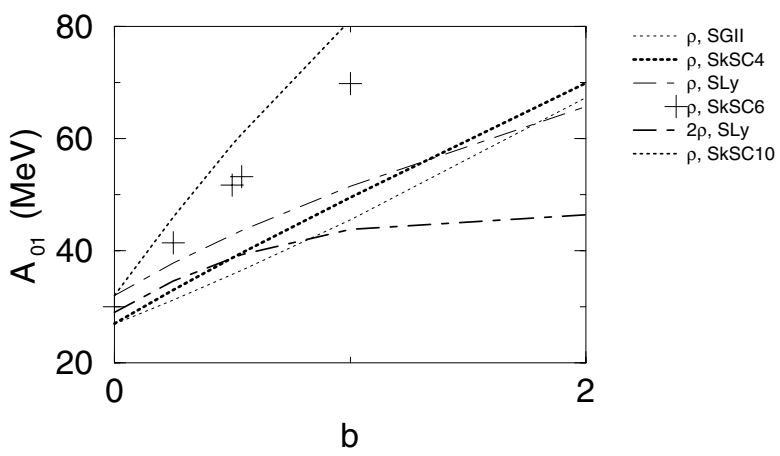

Figure 1. Neutron-proton symmetry energy coefficient $A_{0,1}=$ $\rho /\left(2 \Pi_{R}^{0,1}\right)$ as a function of the asymmetry coefficient $b$ for several Skyrme forces: dotted line (SGII), thick dotted (SkSC4), longshort dashed (SLy(b)), crosses (SkSC6), thick long-short dashed (SLy(b) at twice $\left.\rho_{0}\right)$, medium thick dotted (SkSC10).

In Figure 2 the spin symmetry energy is shown as a function of $b$ with forces SLy, SkSC4, SkSC6 and SkSC10. The values for symmetric nuclear matter are very different. The common trend is the increase of $A_{1,0}$ with $b$, i.e., at very asymmetric matter the spin interaction tends to become more repulsive. However the particular behavior of the spin s.e.c. with $b$ is different for each effective force at a given density. We can compare our results to the ratio of spin susceptibility of interacting neutron matter to the non interacting Fermi gas obtained by Fantoni, Sarsa and Schmidt [14] by means of the auxiliary field diffusion Monte Carlo method. This ratio is proportional to the polarizability as obtained in expression (1) and therefore inversely proportional to the spin symmetry coefficient $A_{1,0}$. First of all we note that, in most cases, the values they find are all positive for the range of densities considered by them, from $0.75 \rho_{0}$ up to $2.5 \rho_{0}$ (in our calculation the total density was kept constant and equal to the saturation density $\rho_{0}$ ). The slope seems to be nearly the same as that we obtain for low values of the n-p asymmetry. Consequently they may obtain instabilities for higher density neutron matter whereas we do not observe this result in our calculations with Skyrme forces at the saturation density (the comparison is meaningful for neutron matter: $b$ very large). A further comparison at different total nuclear densities is to be shown elsewhere [5].

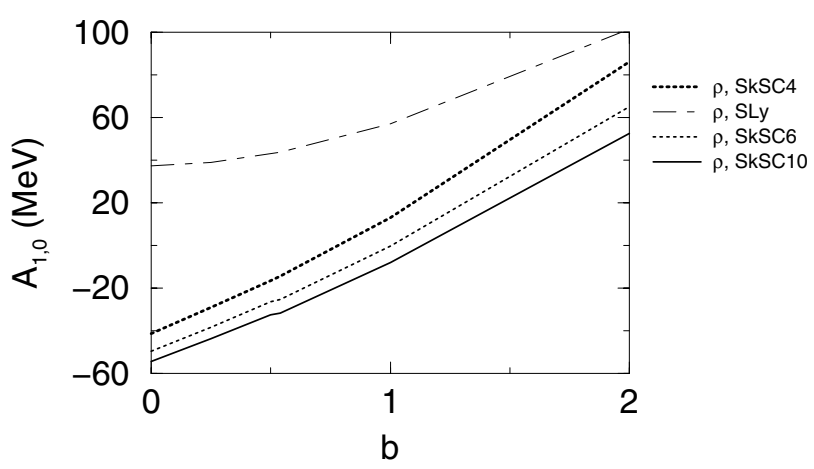

Figure 2. Spin symmetry energy coefficient $A_{1,0}=\rho /\left(2 \Pi_{R}^{1,0}\right)$ as a function of the asymmetry coefficient $b$ for the different forces: thick dotted (SkSC4), long-short dashed (SLy(b)), medium thick dotted (SkSC6), thick solid line (SkSC10).

\section{Summary and Conclusions}

Summarizing, the dependence of the s.e.c. on the n-p asymmetry was studied extending the results of ref. [5]. Different prescriptions for the density fluctuations (expressions $(2,7))$ in asymmetric nuclear matter lead to different dependences of the symmetry energy coefficients on the asymmetry parameter $b$. The n-p asymmetry dependence of the s.e.c. in the different channels was analyzed for different Skyrme forces. They may yield very different behaviors including the possibility (or not) of nuclear matter to undergo phase transitions. These forces can describe different behaviors of the symmetry energy coefficients. Therefore, in principle, different values can be expected for the (bulk) symmetry energy coefficients in asymmetric nuclear matter with different $\mathrm{n}$-p asymmetries. It would be interesting to apply the results obtained here and in [5] in studies of the equation of state of asymmetric nuclear matter, trying to extract experimental constraints for $\mathcal{A}_{s, t}$, in particular in the isospin channel $(s=0, t=1)$. (For works which can be related with the present ideas see, for example, these proceedings - work by W. Lynch - and [15]). Although we have dealt with neutronproton density asymmetry we can expect that the same kind of ideas can be applied for the neutron-proton number asymmetry used in mass formulas for finite nuclei [3]. In the spin channel it is possible to expect spin polarized asymmetric matter yielding magnetic fields in neutron stars, as discussed in [9]. However with the increase of $b$ we find that the spin interaction may be rather repulsive, hindering this magnetization effect with the use of these Skyrme forces for the value of density analyzed here $\left(\rho_{0}\right)$. In this work we do not include the simultaneous study of the density dependence of the symmetry coefficients.

\section{Acknowledgement}

This work was supported by FAPESP, Brazil. The author thanks D. Vautherin for discussions. 


\section{References}

[1] J. Jänecke and E. Comay, Nucl. Phys. A 436, 108 (1985).

[2] C.-H. Lee, T.T.S. Kuo, G.Q. Li, and G.E. Brown, Phys. Rev. C 57, 3488 (1998).

[3] P. Moeller, J.R. Nix, W.D. Myers and W.J. Swiatecki, At. Data Nucl. Data Tables 59, 185 (1995); Y. Aboussir, J.M. Pearson, A.K. Dutta and F. Tondeur, At. Data Nucl. Data Tables 61, 127 (1995). W.D. Myers and W.J. Swiatecki, Nucl. Phys. A601, 141 (1996).

[4] F.L. Braghin, Phys. Lett. B 446, 1 (1999); Nucl. Phys. A 665 , 13 (2000).

[5] F.L. Braghin, Nuc. Phys. A 696, 413 (2001); F.L.B., Erratum Nuc. Phys. A 709, 487 (2002).

[6] R.F. Sawyer, Phys. Rev. C11, 2740 (1975), N. Iwamoto and C.J. Pethick, Phys. Rev. D25, 313 (1982).

[7] J. Navarro, E.S. Hernandez, D. Vautherin, Phys. Rev. C60 (04)5801 (1999).
[8] S. Reddy, M. Prakash, J.M. Lattimer, J.A. Pons, Phys. Rev. C59, 2888 (1999).

[9] M. Kutschera and W. Wójcik, Phys. Lett. B223, 11 (1989).

[10] F.L. Braghin, D. Vautherin and A. Abada, Phys. Rev. C 52, 2504 (1995).

[11] E. Chabanat, P. Bouche, P. Haensel, J. Meyer, and R. Schaeffer, Nucl. Phys. A 627, 710 (1997).

[12] A.D. Dutta, J.-P. Arcoragi, J.M. Pearson, R. Behrman, F. Tondeur, Nucl. Phys. A 458, 77 (1986); F. Tondeur, A.K. Dutta, J.M. Pearson, and R. Behrman, Nucl. Phys. A470, 93 (1987), Y. Aboussir, J.M. Pearson, and F. Tondeur, Nucl. Phys. A549, 155 (1992).

[13] M. Onsi, H. Przysiezniak and J.M. Pearson, Phys. Rev. C50, 460 (1994).

[14] S. Fantoni, A. Sarsa, K.E. Schmidt, Phys. Rev. Lett. 87, 181101 (2001)

[15] Bao-An Li, Nucl. Phys. A708, 365 (2002). 